\title{
Prebiotic, Probiotic, and Synbiotic Supplementation in Chronic Kidney Disease: A Systematic Review and Meta-analysis
}

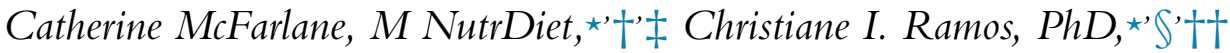

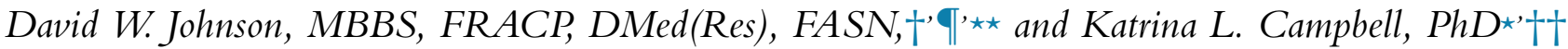

Objective: Gut dysbiosis has been implicated in the pathogenesis of chronic kidney disease (CKD). Restoring gut microbiota with prebiotic, probiotic, and synbiotic supplementation has emerged as a potential therapeutic intervention but has not been systematically evaluated in the CKD population.

Design and Methods: This is a systematic review. A structured search of MEDLINE, CINAHL, EMBASE, Cochrane Central Register of Controlled Trials, and the International Clinical Trials Register Search Portal was conducted for articles published since inception until July 2017. Included studies were randomized controlled trials investigating the effects of prebiotic, probiotic, and/or synbiotic supplementation ( $>1$ week) on uremic toxins, microbiota profile, and clinical and patient-centered outcomes in adults and children with CKD.

Results: Sixteen studies investigating 645 adults met the inclusion criteria; 5 investigated prebiotics, 6 probiotics, and 5 synbiotics. The quality of the studies (Grades of Recommendation, Assessment, Development and Evaluation) ranged from moderate to very low. Prebiotic, probiotic, and synbiotic supplementation may have led to little or no difference in serum urea (9 studies, 345 participants: mean difference $[\mathrm{MD}]-0.30 \mathrm{mmol} / \mathrm{L}, 95 \%$ confidence interval $[\mathrm{Cl}]-2.20$ to $\left.1.61, P=.76, \mathrm{l}^{2}=53 \%\right)$, indoxyl sulfate $(4$ studies, 144 participants: $\mathrm{MD}-0.02 \mathrm{mg} / \mathrm{dL}, 95 \% \mathrm{Cl}-0.09$ to $0.05, P=.61, \mathrm{l}^{2}=0 \%$ ), and $\mathrm{p}$-cresyl sulfate (4 studies, 144 participants: MD $-0.13 \mathrm{mg} / \mathrm{dL}, 95 \% \mathrm{Cl}-0.41$ to $0.15, P=.35, \mathrm{I}^{2}=0 \%$ ). Prebiotic supplementation may have slightly reduced serum urea concentration (4 studies, 105 participants: MD $-2.23 \mathrm{mmol} / \mathrm{L}, 95 \% \mathrm{Cl}-3.83$ to $-0.64, P=.006, \mathrm{I}^{2}=11$ ). Of the 2 studies investigating microbiota changes, synbiotic interventions significantly increased Bifidobacterium. Supplement effects on clinical outcomes were uncertain.

Conclusions: There is limited evidence to support the use of prebiotics, probiotics, and/or synbiotics in CKD management. (c) 2018 by the National Kidney Foundation, Inc. All rights reserved.

\section{Introduction}

$\mathrm{C}$ HRONIC KIDNEY DISEASE (CKD) is a major health burden worldwide. ${ }^{1}$ The prevalence of CKD is steadily increasing, ${ }^{2}$ and individuals with CKD have a significantly increased risk of cardiovascular disease, ${ }^{3}$ which is only partially explained by the traditional risk factors of older age, obesity, tobacco use, diabetes mellitus, hypertension, and dyslipidemia. ${ }^{4}$
Over the last decade, there has been a growing body of evidence linking gut dysbiosis and intestinal wall permeability to progressive kidney failure and cardiovascular risk $^{5,6}$ via systemic inflammation and production of uremic toxins, including indoxyl sulfate (IS), p-cresyl sulfate (PCS), and trimethylamine $\mathrm{N}$-oxide (TMAO). The imbalance of gut microorganisms in patients with CKD may be therapeutically modifiable. For example,

\footnotetext{
${ }^{\star}$ Faculty of Health Sciences and Medicine, Bond University, Gold Coast, Queensland, Australia.

${ }^{\dagger}$ School of Medicine, University of Queensland, Brisbane, Queensland, Australia.

${ }^{\ddagger}$ Renal Department, Sunshine Coast University Hospital, Birtinya, Queensland, Australia.

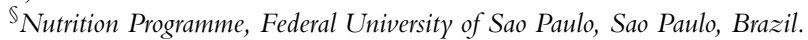

"Department of Nephrology, Princess Alexandra Hospital, Brisbane, Queensland, Australia.

${ }^{\star \star}$ Translational Research Institute, Brisbane, QLD, Australia.

${ }^{{ }^{\dagger}}$ Department of Nutrition and Dietetics, Princess Alexandra Hospital, Brisbane, Queensland, Australia.

Financial Disclosure: The authors declare that they have no relevant financial interests.
} 
prebiotics, probiotics, and synbiotics may competitively decrease the relative population of protein-fermenting intestinal flora and consequently reduce the production of uremic toxins. This is achieved by altering the carbohydrate-to-protein ratio and augmenting shortchain fatty-acid production. This low-cost therapy, therefore, represents an appealing therapeutic strategy.

To date, the results from individual studies investigating the effects of prebiotic, probiotic, and/or synbiotic supplementation within the CKD population have produced conflicting results. ${ }^{10-12}$ Furthermore, there has been no prior systematic review of the effects of prebiotic, probiotic, and/or synbiotic supplementation on kidney function, uremic toxin production, microbiota composition, and patient-level outcomes exclusively within the CKD population. Therefore, the aim of this review was to systematically evaluate randomized controlled trials (RCTs) assessing the effectiveness of prebiotic, probiotic, and/or synbiotic supplementation on clinical and patient-centered outcomes in CKD.

\section{Materials and Methods}

This review was conducted according to the Preferred Reporting Items for Systematic Reviews and Meta-Analyses reporting guidelines following a prespecified protocol (PROSPERO CRD42017075771).

\section{Criteria for Considering Studies}

Studies were included in the review if they met all the following criteria: (1) RCT including crossover, cluster, or quasi-RCT designs; (2) in participants with CKD as defined by the Kidney Disease Outcome Quality Initiative Guidelines ${ }^{14}$; and (3) interventions that included supplementation with prebiotics, probiotics, or synbiotics for a duration of at least 1 week. Studies were excluded if they were using high-dose prebiotics for purgation, populations with altered gastrointestinal function, and those where nutrition was provided enterally.

The primary outcome was change in kidney function (estimated glomerular filtration rate [eGFR], serum creatinine) and kidney damage (proteinuria, albuminuria). Secondary outcomes included uremic toxins (urea, free and protein-bound concentrations of serum IS and PCS, TMAO phenylacetylglutamine [PAG]), microbiota composition, change in clinical markers (fasting blood glucose, $\mathrm{HbA} 1 \mathrm{c}$, weight, waist circumference), markers of cardiovascular risk (left ventricular mass index, diastolic function, blood pressure, blood lipid profile), infections including antibiotic use, clinical outcomes (hospitalizations, cardiovascular events, progression to end-stage kidney disease [ESKD], mortality, adverse events), and patient-centered outcomes (colonic transit time, fecal characteristics, gastrointestinal tolerance, health-related quality of life and treatment adherence).

\section{Search Methods}

A comprehensive search was conducted using MEDLINE, CINAHL, Embase, and Cochrane databases from inception until 2017 using a combination of $\mathrm{MeSH}$ and free-text terms relevant to the review in consultation with an experienced systematic review search librarian. $\mathrm{MeSH}$ search terms are outlined in the prespecified protocol (PROSPERO CRD42017075771). Searches of the International Clinical Trials Register and clinicaltrials.gov were undertaken to identify any ongoing trials. In addition, manual searches were performed to retrieve relevant studies through citation searches of conference abstracts, theses, and biographies of relevant published articles.

\section{Data Collection}

Articles were screened independently by 2 review authors (C.M., C.I.R.), with disagreements resolved by consensus or discussion with a third reviewer (K.L.C.). Data extraction from included studies comprised intervention details, study design, duration, sample size, attrition, and participant characteristics. Mean, standard deviations, standard error, or $95 \%$ confidence intervals (CIs) for all prespecified primary and secondary outcome data that were reported at baseline and follow-up were extracted for data analysis. The units of measurement for uremic toxins were converted to $\mathrm{mg} / \mathrm{dL}$ using molecular weights from the Human Metabolome Database. ${ }^{15}$ Corresponding authors were contacted for information that was not published, including missing numerical data of outcome measures.

\section{Assessment of Study and Evidence Quality}

Risk of bias was assessed by 2 review authors (C.M., C.I.R.) independently using Cochrane methodology. ${ }^{16}$ The Grades of Recommendation, Assessment, Development and Evaluation (GRADE) approach for grading the evidence was applied for each outcome category ${ }^{17}$ and assessed independently by 2 review authors (C.M., C.I.R.). Disagreements in risk of bias and GRADE classification were managed by consensus and discussion with a third reviewer (K.L.C.).

\section{Statistical Analysis}

The overall treatment effects for primary and secondary outcomes were calculated as the differences between the intervention and comparison groups' change scores from baseline to the end of follow-up. If the change from baseline score was not available, the end of intervention value was extracted with the assumption that no significant differences were observed at baseline between intervention and comparison groups.

Quantitative analysis was undertaken for adequately reported outcomes by pooling data into Revman (Review Manager 5, version 5.3, the Cochrane Collaboration) for meta-analysis through DerSimonian-Laird random-effects model. ${ }^{16}$ Heterogeneity between studies was assessed using 


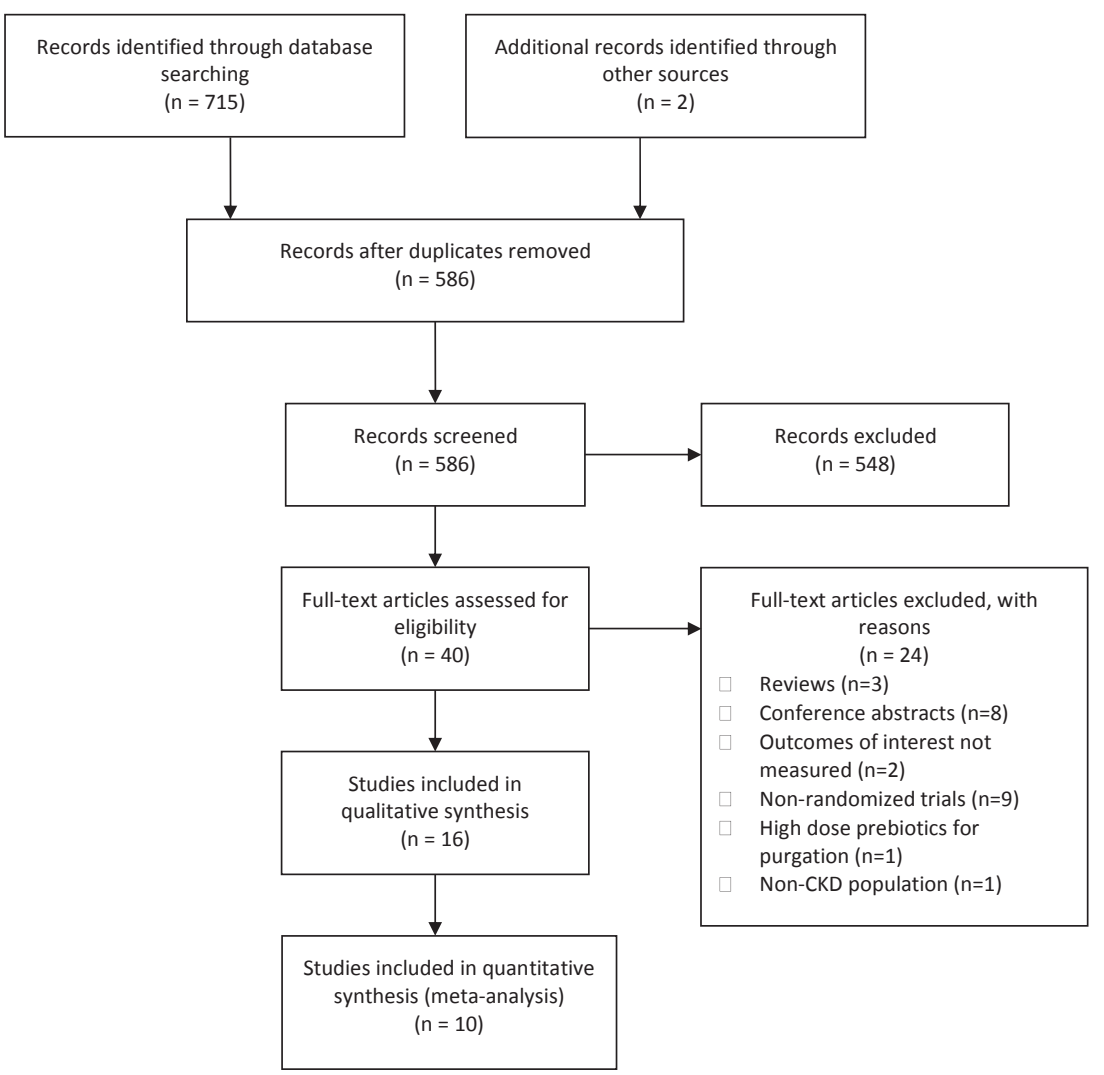

Figure 1. Flowchart describing prebiotic, probiotic, and synbiotic study selection.

the $\mathrm{I}^{2}$ statistic and was considered substantial if the $\mathrm{I}^{2}$ statistic was $\geq 50 \%$. Publication bias was investigated using funnel plots and Egger's test.

\section{Results \\ Characteristics of Included Studies}

The search identified 586 citations after removal of duplicates (Fig. 1). Sixteen studies were included in the review, with a total sample size of 645 participants (individual studies ranging from 9 to 124 participants) and intervention durations ranging from 2 to 24 weeks. All included studies involved adult participants with CKD with 7 studies involving nondialysis patients, ${ }^{7,9,18-23} 8$ studies involving patients undergoing hemodialysis for ESKD, ${ }^{8,24-29}$ and 1 study involving participants undergoing peritoneal dialysis. ${ }^{30}$ Five trialled prebiotics, $^{7,8,18,23,29} 6$ trialled probiotics, $^{21,22,24,26,27,30}$ and 5 trialled synbiotics. $9,19,20,25,28$ (Table 1). Prebiotic, probiotic, and synbiotic formulations are outlined in Table 1. Additional information was provided by 3 of the 9 contacted authors. ${ }^{7,9,19}$ The quality of the studies (GRADE) ranged from moderate to very low (Table 2).

\section{Risk of Bias}

Risk of bias was low in the following domains: detection bias (13 studies), ${ }^{7-9,18-22,24-26,28,30}$ attrition bias (9 studies), $7,9,18,20,21,23,26-28 \quad$ performance bias (7 studies), 7,9,19,20,24,28,30 selection bias (random sequence generation $[5 \text { studies }]^{8,9,20,27,30}$ allocation concealment [6 studies] ${ }^{7,9,20,24,27,30}$ ), and reporting bias (4 studies). ${ }^{7-9,27}$ Five studies were rated as having a high risk of attrition bias (through high loss to follow-up and no explanation of how data were addressed), ${ }^{8,22,24,29,30} 4$ studies were rated as having a high risk of performance bias (no blinding or blinding may have been broken), 8,18,23,29 while 3 were rated as having a high risk of reporting bias (a number of outcome measures were not reported in the results). ${ }^{21,22,26}$ Bias related to control of dietary intake was low in 4 studies, 9,23,28,29 while 4 studies were rated as having an unclear risk of bias related to dietary advice and assessment of dietary compliance. ${ }^{7,18,27,30}$ The 8 studies which did not provide dietary advice nor assess dietary intake were rated as having a high risk of bias related to potential dietary confounders. $8,19-22,24-26$ Three studies were determined as having other risks of bias relating to the absence of a washout period in crossover trials and possible or undeclared conflicts of interest ${ }^{18,21,22}$ (Fig. 2).

\section{Change in Kidney Function and Damage}

Nutrition supplementation probably made little or no difference to kidney function, measured by eGFR (3 studies, 132 participants: mean difference [MD] $0.34 \mathrm{~mL} /$ $\min / 1.73 \mathrm{~m}^{2}, 95 \% \mathrm{CI}-2.20$ to $2.89, P=.79, \mathrm{I}^{2}=0 \%$; 


\begin{tabular}{|c|c|c|c|c|c|c|c|c|}
\hline \multirow[b]{2}{*}{ Author, year } & \multirow[b]{2}{*}{ Study Design } & \multirow[b]{2}{*}{ Participants } & \multirow[b]{2}{*}{ Intervention } & \multirow[b]{2}{*}{ Time } & \multirow[b]{2}{*}{ Primary Outcome Measure } & \multicolumn{3}{|c|}{ Potential Confounders } \\
\hline & & & & & & $\begin{array}{l}\text { Assessed } \\
\text { Diet }\end{array}$ & $\begin{array}{l}\text { Washout } \\
\text { Period }\end{array}$ & Compliance \\
\hline Bliss, 1996 & $\begin{array}{l}\text { Single-blind, crossover } \\
\text { RCT }\end{array}$ & $\begin{array}{l}\mathrm{n}=16(63 \% \text { male }) \\
\text { nondialysis }\end{array}$ & Prebiotic: $50 \mathrm{~g}$ gum arabic & $1 \mathrm{wk}$ & $\begin{array}{l}\text { Stool weight, bacterial } \\
\text { mass, fecal } N \text {, urinary } N\end{array}$ & No & No & No \\
\hline Younes, 2006 & Crossover RCT & $\begin{array}{c}\mathrm{n}=9(67 \% \text { male }) \\
\text { nondialysis }\end{array}$ & $\begin{array}{l}\text { Prebiotic: } 40 \mathrm{~g} \\
\quad \text { Fermentable } \mathrm{CHO}\end{array}$ & 2 wk & $\begin{array}{l}\text { Fecal } \mathrm{N} \text { and stool weight, } \\
\text { urinary } \mathrm{N}\end{array}$ & No & Yes & Yes \\
\hline Sirich, 2014 & Single-blind RCT & $\begin{array}{c}\mathrm{n}=40(60 \% \text { male }) \\
\text { hemodialysis }\end{array}$ & $\begin{array}{l}\text { Prebiotic: } 30 \mathrm{~g} \mathrm{Hi}-\text { maize } \\
\quad 260\end{array}$ & $6 \mathrm{wk}$ & $\begin{array}{l}\text { Free and total plasma IS } \\
\text { and PCS }\end{array}$ & No & NA & Yes \\
\hline Xie, 2015 & $\mathrm{RCT}$ & $\begin{array}{l}\mathrm{n}=124(55 \% \text { male }) \\
\text { hemodialysis }\end{array}$ & $\begin{array}{l}\text { Prebiotic: } 10 \mathrm{~g} \text { or } 20 \mathrm{~g} \\
\text { soluble dietary fiber }\end{array}$ & $6 \mathrm{wk}$ & $\begin{array}{l}\text { Lipid profile, oxidative and } \\
\text { inflammatory status }\end{array}$ & Yes & NA & No \\
\hline Poesen, 2016 & $\begin{array}{l}\text { Double-blind crossover } \\
\text { RCT }\end{array}$ & $\begin{array}{l}\mathrm{n}=40(70 \% \text { male }) \text { CKD } \\
\text { stage: } 3 \mathrm{~b}, 4\end{array}$ & $\begin{array}{l}\text { Prebiotic: } 20 \mathrm{~g} \\
\quad \text { arabinoxylan } \\
\text { oligosaccharides (AXOS) }\end{array}$ & 4 wk & $\begin{array}{l}\text { TMAO, PCS, PCG, IS, } \\
\text { PAG, urea }\end{array}$ & Yes & Yes & Yes \\
\hline $\begin{array}{l}\text { Ranganathan, } \\
2009 \text { (pilot) }\end{array}$ & $\begin{array}{l}\text { Double-blind, crossover } \\
\text { RCT }\end{array}$ & $\begin{array}{l}\mathrm{n}=13(69 \% \text { male }) \text { CKD } \\
\text { stage: } 3,4\end{array}$ & $\begin{array}{l}\text { Probiotic: } 9 \times 10^{10} \mathrm{CFU} / \\
\text { day (Kibow Biotics }{ }^{\circledR} \text { : } \\
\text { S. thermophilus KB19, } \\
\text { L. acidophilus KB27, and } \\
\text { B. longum KB31) }\end{array}$ & $3 \mathrm{mo}$ & $\begin{array}{l}\text { BUN, uric acid, serum } \\
\text { creatinine or CRP; Gut } \\
\text { microbiota }\end{array}$ & No & No & No \\
\hline $\begin{array}{l}\text { Ranganathan, } \\
2010 \text { (pilot) }\end{array}$ & $\begin{array}{l}\text { Double-blind, crossover } \\
\text { RCT }\end{array}$ & $\begin{array}{l}\mathrm{n}=46(67 \% \text { male }) \text { CKD } \\
\text { stage: } 3,4\end{array}$ & $\begin{array}{l}\text { Probiotic: } 9 \times 10^{10} \mathrm{CFU} / \\
\text { day (Kibow Biotics) }\end{array}$ & $3 \mathrm{mo}$ & $\begin{array}{l}\text { BUN, serum creatinine, uric } \\
\text { acid QoL }\end{array}$ & No & No & No \\
\hline Natarajan, 2014 & $\begin{array}{l}\text { Double-blind, crossover } \\
\text { RCT }\end{array}$ & $\begin{array}{l}\mathrm{n}=22(27 \% \text { male }) \\
\text { hemodialysis }\end{array}$ & $\begin{array}{l}\text { Probiotic: } 18 \times 10^{10} \mathrm{CFU} / \\
\text { day (Renadyl }{ }^{\circledR}: S . \\
\text { thermophilus KB19, } \\
\text { L. acidophilus KB27, and } \\
\text { B. longum KB31) }\end{array}$ & $2 \mathrm{mo}$ & $\begin{array}{l}\text { Indoxyl glucuronide, IS, } \\
\text { PCS, IAA, hippuric acid; } \\
\text { Quality of life }\end{array}$ & No & Yes & Yes \\
\hline Wang, 2015 & Double-blind, RCT & $\begin{array}{l}\mathrm{n}=39(46 \% \text { male }) \\
\text { peritoneal dialysis }\end{array}$ & $\begin{array}{l}\text { Probiotic: } 4 \times 10^{9} \mathrm{CFU} / \text { day } \\
\text { (B. bifidum A218, } \\
\text { B. catenulatum A302, } \\
\text { B. longum A101, } \\
\text { L. platarum A87) }\end{array}$ & $6 \mathrm{mo}$ & Endotoxin & No & NA & Yes \\
\hline Soleimani, 2016 & Double-blind, RCT & $\begin{array}{l}\mathrm{n}=60(67 \% \text { male }) \\
\quad \text { diabetic } \\
\text { hemodialysis }\end{array}$ & $\begin{array}{l}\text { Probiotic: } 6 \times 10^{9} \text { CFU/day } \\
\text { (L. acidophilus, L. casei, } \\
\text { and B. bifidum) }\end{array}$ & $3 \mathrm{mo}$ & Glucose homeostasis & Yes & NA & Yes \\
\hline Borges, 2016 & Double-blind, RCT & $\begin{array}{c}\mathrm{n}=33(64 \% \text { male }) \\
\text { hemodialysis }\end{array}$ & $\begin{array}{l}\text { Probiotic: } 90 \times 10^{9} \mathrm{CFU} / \\
\text { day (S. thermophilus, } \\
\text { L. acidophilus, } \\
\text { B. longum) }\end{array}$ & $3 \mathrm{mo}$ & $\begin{array}{l}\text { Serum urea, CRP, IL-6, IS, } \\
\text { PCS, IAA fecal bacterial } \\
\text { profile }\end{array}$ & No & NA & Yes \\
\hline Cruz-Mora, 2014 & Double-blind, RCT & $\begin{array}{l}\mathrm{n}=18(83 \% \text { male }) \\
\text { hemodialysis }\end{array}$ & $\begin{array}{l}\left.\text { Synbiotic (Nutrihealth }{ }^{\circledR}\right) \text { : } \\
\text { - } \\
\text { Prebiotic: } 2.3 \mathrm{~g} / \text { day } \\
\text { (Inulin) } \\
\text { - } \\
\text { Probiotic: } \\
2 \times 10^{12} \text { CFU/day (L. } \\
\text { acidophilus \& B. } \\
\text { bifidum) }\end{array}$ & $2 \mathrm{mo}$ & Gut microbiota & No & NA & No \\
\hline
\end{tabular}


- Prebiotic: 6.6 g/day

$$
\text { (inulin) }
$$

- Probiotic:

$5,7 \times 10^{10} \mathrm{CFU} /$ day

(L. plantarum, L. casei

subsp. Rhamnosus,

L. gasseri, B. infantis,

B. longum, L. acidophi-

lus, L. salivarius,

L. sporogenes, $S$.

thermophilus)

\begin{tabular}{|c|c|c|c|c|c|c|c|c|}
\hline $\begin{array}{l}\text { Viramontes- } \\
\text { Horner, } 2015\end{array}$ & Double-blind, RCT & $\begin{array}{c}\mathrm{n}=35(91 \% \text { male }) \\
\text { hemodialysis }\end{array}$ & $\begin{array}{l}\text { Synbiotic (Nutrihealth }{ }^{\circledR} \text { ): } \\
\text { - Prebiotic: } 2.3 \mathrm{~g} / \text { day } \\
\text { (inulin) } \\
\text { - Probiotic: } \\
11 \times 10^{6} \mathrm{CFU} / \text { day } \\
\text { (L. acidophilus NCFM } \\
\text { and B. lactis Bi-07) } \\
\text { - Plus: omega-3 and } \\
\text { vitamins }\end{array}$ & $2 \mathrm{mo}$ & Gl symptoms & Yes & NA & No \\
\hline Dehghani, 2016 & Double-blind, RCT & $\begin{array}{l}\mathrm{n}=66(76 \% \text { male }) \mathrm{CKD} \\
\text { stage: } 3,4\end{array}$ & $\begin{array}{l}\text { Synbiotic (Familact }{ }^{\circledR} \text { ) } \\
\text { - } \quad \text { Prebiotic (FOS) } \\
\text { - } \text { Probiotic: (L. casei, } \\
\text { L. acidophilus, L. bul- } \\
\text { garicus, L. rhamnosus, } \\
\text { B. breve, B. longum, } \\
\text { S. thermophilus) }\end{array}$ & 6 wk & $\begin{array}{l}\text { BUN, serum creatinine, uric } \\
\text { acid, } \mathrm{CrCl} \text {, eGFR }\end{array}$ & No & NA & No \\
\hline Rossi, 2016 & $\begin{array}{l}\text { Double-blind, crossover } \\
\text { RCT }\end{array}$ & $\begin{array}{c}n=21(62 \%) \text { CKD stage: } 4 \\
5 \text { nondialyzed }\end{array}$ & $\begin{array}{l}\text { Synbiotic: } \\
\text { - Prebiotic: } 15 \text { g/day } \\
\text { (inulin, FOS, and GOS) } \\
\text { - Probiotic: } \\
9 \times 10^{10} \text { CFU/day (9 } \\
\text { strains from Lactoba- } \\
\text { cillus, Bifidobacteria, } \\
\text { and Streptococcus } \\
\text { genera) }\end{array}$ & $6 \mathrm{wk}$ & Serum IS & Yes & Yes & Yes \\
\hline
\end{tabular}

BUN, blood urea nitrogen; CFU, colony-forming unit; CHO, carbohydrate; CKD, chronic kidney disease; CRP, c-reactive protein; FOS, fructooligosaccharide; eGFR, estimated glomerular filtration rate; GI, gastrointestinal; GOS, galactooligosaccharide; HDL-C, high-density lipoprotein cholesterol; IAA, indole 3-acetic acid; IL, interleukin; IS, indoxyl sulfate; LDL-C, low-density lipoprotein cholesterol; N, nitrogen NCFM, North Carolina Food Microbiology; PCS, p-cresyl sulfate; PAG, phenylacetylglutamine; PCG, $p$-cresyl glucuronide; TC, total cholesterol; TGs, triglycerides; TMAO, trimethylamine $\mathrm{N}$-oxide; TNF- $\alpha$, tumor necrosis factor $\alpha$. 
Table 2. Grading of Recommendations, Assessment, Development and Evaluation (GRADE) Assessment of Prebiotic, Probiotic, and Synbiotic Supplementation Compared With Placebo in Patients With Chronic Kidney Disease

\begin{tabular}{|c|c|c|c|c|c|c|c|c|c|c|c|c|}
\hline \multicolumn{7}{|c|}{ Certainty Assessment } & \multicolumn{4}{|r|}{ Effect } & \multirow[b]{2}{*}{ Certainty } & \multirow[b]{2}{*}{ Importance } \\
\hline $\begin{array}{l}\text { No. of } \\
\text { Studies }\end{array}$ & Study Design & Risk of Bias & Inconsistency & Indirectness & Imprecision & $\begin{array}{c}\text { Other } \\
\text { Considerations }\end{array}$ & \multicolumn{2}{|c|}{ No. of Patients } & $\begin{array}{l}\text { Relative } \\
\text { (95\% Cl) }\end{array}$ & $\begin{array}{l}\text { Absolute } \\
(95 \% \mathrm{Cl})\end{array}$ & & \\
\hline \multicolumn{13}{|c|}{ Urea (follow-up: range 4-24 weeks; assessed with: $\mathrm{mmol} / \mathrm{L}$ ) } \\
\hline 9 & $\begin{array}{l}\text { Randomized } \\
\text { trials }\end{array}$ & Serious ${ }^{\star}, \dagger, \neq, \S$ & $\begin{array}{l}\text { Not } \\
\text { serious }\end{array}$ & $\begin{array}{l}\text { Not } \\
\text { serious }\end{array}$ & Serious $\|$ & None & 203 & 200 & - & $\begin{array}{l}\text { MD } 0.3 \text { lower ( } 2.2 \text { lower to } \\
1.61 \text { higher) }\end{array}$ & $\begin{array}{c}\oplus \oplus \bigcirc \bigcirc \\
\text { Low }\end{array}$ & $\begin{array}{l}\text { Not } \\
\text { important }\end{array}$ \\
\hline \multicolumn{13}{|c|}{ p-cresyl sulfate (follow-up: range 4-24 weeks; assessed with: $\mathrm{mg} / \mathrm{dL}$ ) } \\
\hline 4 & $\begin{array}{l}\text { Randomized } \\
\text { trials }\end{array}$ & Serious $\$$ & $\begin{array}{l}\text { Not } \\
\text { serious }\end{array}$ & $\begin{array}{l}\text { Not } \\
\text { serious }\end{array}$ & Serious $\|$ & None & 97 & 98 & - & $\begin{array}{l}\text { MD } 0.13 \text { lower ( } 0.41 \text { lower } \\
\text { to } 0.15 \text { higher) }\end{array}$ & $\begin{array}{l}\oplus \oplus \bigcirc \bigcirc \\
\text { Low }\end{array}$ & $\begin{array}{l}\text { Not } \\
\text { important }\end{array}$ \\
\hline \multicolumn{13}{|c|}{ Indoxyl sulfate (follow-up: range 4-24 weeks; assessed with: $\mathrm{mg} / \mathrm{dL}$ ) } \\
\hline 4 & $\begin{array}{l}\text { Randomized } \\
\text { trials }\end{array}$ & Serious $\$$ & Not serious & $\begin{array}{l}\text { Not } \\
\text { serious }\end{array}$ & Serious $\llbracket$ & None & 97 & 98 & - & $\begin{array}{l}\text { MD } 0.02 \text { lower ( } 0.09 \text { lower } \\
\text { to } 0.05 \text { higher) }\end{array}$ & $\begin{array}{l}\oplus \oplus \bigcirc \bigcirc \\
\text { Low }\end{array}$ & $\begin{array}{l}\text { Not } \\
\text { important }\end{array}$ \\
\hline \multicolumn{13}{|c|}{ eGFR (follow-up: range 4-6 weeks; assessed with: $\mathrm{mL} / \mathrm{min} / 1.73 \mathrm{~m}^{2}$ ) } \\
\hline 3 & $\begin{array}{l}\text { Randomized } \\
\text { trials }\end{array}$ & Not serious & Not serious & $\begin{array}{l}\text { Not } \\
\text { serious }\end{array}$ & Serious $\|$ & None & 92 & 96 & - & $\begin{array}{l}\text { MD } 0.34 \text { higher ( } 2.2 \text { lower } \\
\text { to } 2.89 \text { higher) }\end{array}$ & $\begin{array}{l}\oplus \oplus \oplus \bigcirc \\
\quad \text { Moderate }\end{array}$ & $\begin{array}{l}\text { Not } \\
\text { important }\end{array}$ \\
\hline \multicolumn{13}{|c|}{ Weight (follow-up: range 4-24 weeks; assessed with: kg) } \\
\hline 7 & $\begin{array}{l}\text { Randomized } \\
\text { trials }\end{array}$ & Serious ${ }^{*}, \dagger,+,{ }^{* *}$ & Not serious & $\begin{array}{l}\text { Not } \\
\text { serious }\end{array}$ & Serious $\|$ & None & 226 & 233 & - & $\begin{array}{l}\text { MD } 0.4 \text { higher }(0.95 \text { lower } \\
\text { to } 1.74 \text { higher) }\end{array}$ & $\begin{array}{c}\oplus \oplus \bigcirc \bigcirc \\
\text { Low }\end{array}$ & $\begin{array}{l}\text { Not } \\
\text { important }\end{array}$ \\
\hline \multicolumn{13}{|c|}{ Blood glucose (follow-up: range 4-12 weeks; assessed with: $\mathrm{mg} / \mathrm{dL}$ ) } \\
\hline 2 & $\begin{array}{l}\text { Randomized } \\
\text { trials }\end{array}$ & Not serious & Serious $+\dagger$ & $\begin{array}{l}\text { Not } \\
\text { serious }\end{array}$ & Serious $\|$ & $\begin{array}{l}\text { Publication } \\
\text { bias strongly } \\
\text { suspected } \neq \neq\end{array}$ & 70 & 70 & - & $\begin{array}{l}\text { MD } 0.33 \text { lower ( } 7.17 \text { lower } \\
\text { to } 6.5 \text { higher) }\end{array}$ & $\begin{array}{l}\oplus \bigcirc \bigcirc \bigcirc \\
\text { Very low }\end{array}$ & $\begin{array}{l}\text { Not } \\
\text { important }\end{array}$ \\
\hline \multicolumn{13}{|c|}{ Triglycerides (follow-up: range 6-12 weeks; assessed with: $\mathrm{mg} / \mathrm{dL}$ ) } \\
\hline 3 & $\begin{array}{l}\text { Randomized } \\
\text { trials }\end{array}$ & Serious ${ }^{*}, \dagger,+,^{* *}$ & Not serious & $\begin{array}{l}\text { Not } \\
\text { serious }\end{array}$ & Serious $\|$ & $\begin{array}{l}\text { Publication } \\
\text { bias strongly } \\
\text { suspected } \neq \neq\end{array}$ & 130 & 133 & - & $\begin{array}{l}\text { MD } 1.57 \text { lower ( } 22.56 \\
\text { lower to } 19.42 \text { higher) }\end{array}$ & $\begin{array}{l}\oplus \bigcirc \bigcirc \bigcirc \\
\text { Very low }\end{array}$ & $\begin{array}{l}\text { Not } \\
\text { important }\end{array}$ \\
\hline \multicolumn{13}{|c|}{ Cholesterol (follow-up: range 6-12 weeks; assessed with: mg/dL) } \\
\hline 3 & $\begin{array}{l}\text { Randomized } \\
\text { trials }\end{array}$ & Serious ${ }^{\star}, \dagger, \neq,{ }^{\star *}$ & Serioust† & $\begin{array}{l}\text { Not } \\
\text { serious }\end{array}$ & Serious $\|$ & None & 130 & 133 & - & $\begin{array}{l}\text { MD } 4.88 \text { lower ( } 14.43 \text { lower } \\
\text { to } 4.68 \text { higher) }\end{array}$ & $\begin{array}{l}\oplus \bigcirc \bigcirc \bigcirc \\
\text { Very low }\end{array}$ & $\begin{array}{l}\text { Not } \\
\text { important }\end{array}$ \\
\hline \multicolumn{13}{|c|}{ LDL cholesterol (follow-up: range 6-12 weeks; assessed with: mg/dL) } \\
\hline 3 & $\begin{array}{l}\text { Randomized } \\
\text { trials }\end{array}$ & Serious $^{*}, \dagger, \pm,{ }^{* *}$ & Serioust† & $\begin{array}{l}\text { Not } \\
\text { serious }\end{array}$ & Serious $\|$ & None & 130 & 133 & - & $\begin{array}{l}\text { MD } 11.76 \text { lower (15.15 lower } \\
\text { to } 8.36 \text { lower) }\end{array}$ & $\begin{array}{l}\oplus \bigcirc \bigcirc \bigcirc \\
\text { Very low }\end{array}$ & $\begin{array}{l}\text { Not } \\
\text { important }\end{array}$ \\
\hline
\end{tabular}

$\mathrm{Cl}$, confidence interval; eGFR, estimated glomerular filtration rate; LDL, low-density lipoprotein; MD, mean difference.

*Insufficient information about sequence generation.

†Insufficient information about allocation concealment.

$¥$ Selective reporting likely.

$\mathbb{S}$ A number of included studies did not control for dietary changes nor assess participants dietary intake.

"Small participant numbers and wide $\mathrm{Cl}$ suggests both benefit and no benefit.

-Small participant numbers.

**Blinding was not adequate.

††Significant heterogeneity $\mathrm{I}^{2}>50 \%$.

$\ddagger \ddagger$ Publication bias suspected. 


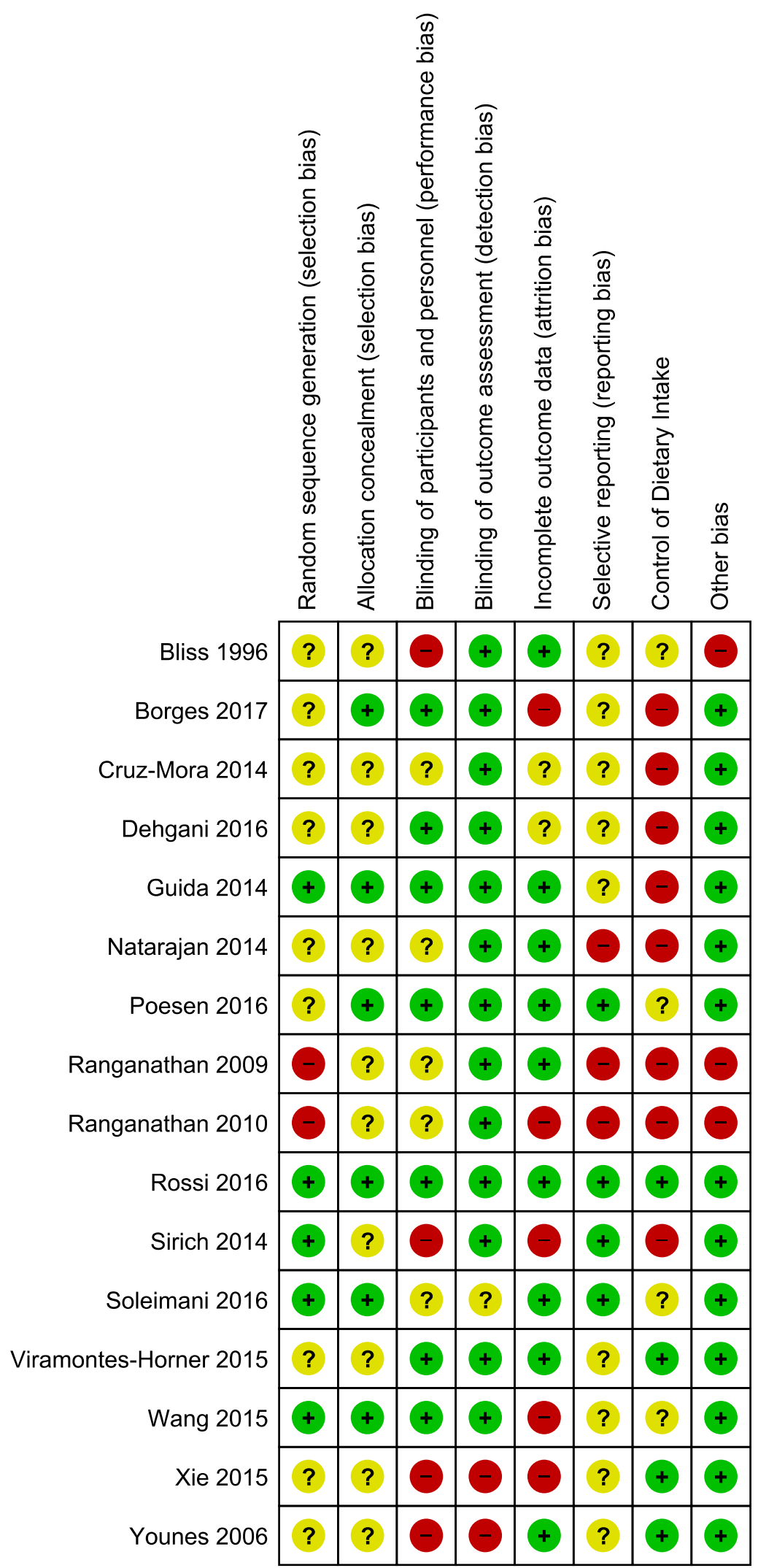

Figure 2. Risk of bias summary across the included studies. Unclear risk of bias: "?", low risk of bias: "+", and high risk of bias: "-". 


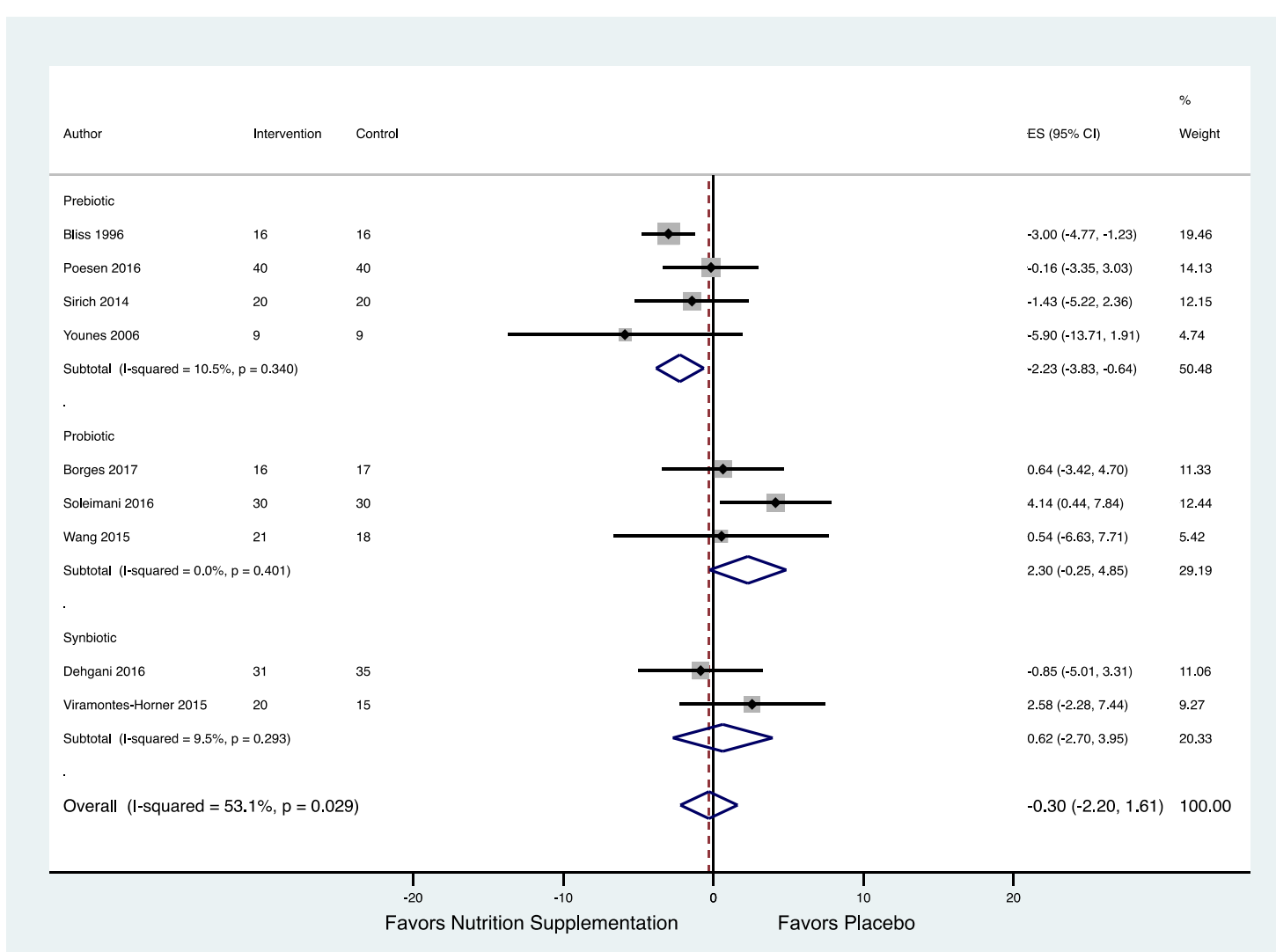

Figure 3. Effects of prebiotic, probiotic, and synbiotic supplementation on serum urea (mmol/L) in patients with CKD. The means and SDs of changes from baseline are reported for trials. Effects of trials are presented as weights (percentages) and mean differences $(95 \%$ Cls). Weights are from random-effects analysis. Cls, confidence intervals; CKD, chronic kidney disease; IV, inverse variance; SDs, standard deviations.

moderate certainty evidence) (Supplementary Fig. 1). ${ }^{7,9,19}$ Two studies reported that nutrition supplementation had no impact on serum creatinine. ${ }^{18,19}$

Kidney damage was reported in one study, with a significant increase in albuminuria $(38 \mathrm{mg} / 24 \mathrm{hr}, 95 \% \mathrm{CI}, 1-$ $295 \mathrm{mg} / 24 \mathrm{hr}, P=.03)$ noted after synbiotic therapy. ${ }^{9}$ The effect of nutrition supplementation on proteinuria was not reported as an outcome measure in any included study.

\section{Uremic Toxins}

Nutrition supplementation may have led to little or no difference to serum urea (9 studies, 345 participants: MD $-0.30 \mathrm{mmol} / \mathrm{L}, 95 \% \mathrm{CI}-2.20$ to $1.61, P=.76$, $\mathrm{I}^{2}=53 \%$; low certainty evidence) $7,8,18,19,23,24,27,28,30$ (Supplementary Fig. 2), serum IS (4 studies, 144 participants: MD $-0.02 \mathrm{mg} / \mathrm{dL}, 95 \% \mathrm{CI}-0.09$ to 0.05 , $P=.61, \mathrm{I}^{2}=0 \%$; moderate certainty evidence), ${ }^{7-9,24}$ and serum PCS (4 studies, 144 participants: MD $-0.13 \mathrm{mg}$ / $\mathrm{dL}, 95 \% \mathrm{CI}-0.41$ to $0.15, P=.35, I^{2}=0 \%$; moderate certainty evidence) $)^{7-9,24}$ (Supplementary Figs. 3 and 4).

Sensitivity analyses were conducted to determine the effects that the type of nutrition supplementation, CKD stage, and duration of intervention had on serum urea levels. Prebiotics may have slightly reduced serum urea concentrations (4 studies, 105 participants: MD $-2.23 \mathrm{mmol} / \mathrm{L}, 95 \% \mathrm{CI}-3.83$ to $-0.64, P=.006$, $\left.\mathrm{I}^{2}=11 \%\right)^{7,8,18,23}$ though not probiotics (3 studies, 132 participants: MD $2.30 \mathrm{mmol} / \mathrm{L}, 95 \% \mathrm{CI}-0.25$ to 4.85 , $\left.P=.08, \mathrm{I}^{2}=0 \%\right)^{24,27,30}$ nor synbiotics (2 studies, 108 participants, MD $0.62 \mathrm{mmol} / \mathrm{L}, 95 \% \mathrm{CI}-2.70$ to 3.95 , $\left.P=.71, \mathrm{I}^{2}=9 \%\right)^{19,28}$ (Fig. 3). A small but statistically significant reduction was observed in serum urea concentration in nondialysis patients (4 studies, 131 participants: $\mathrm{MD}-2.12 \mathrm{mmol} / \mathrm{L}, 95 \% \mathrm{CI}-3.86$ to $-0.37, P=.02$, $\left.\mathrm{I}^{2}=17 \%\right)^{7,18,19,23}$ but not in dialysis patients ( 5 studies, 214 participants: MD $1.36 \mathrm{mmol} / \mathrm{L}, 95 \% \mathrm{CI}-0.76$ to 3.48, $\left.P=.21, \mathrm{I}^{2}=14 \%\right)^{8,24,27,28,30}$ (Fig. 4). There was no subgroup difference by intervention duration.

Sensitivity analyses demonstrated no subgroup differences by type of nutrition supplementation, CKD stage, or intervention duration for both IS and PCS.

One study reported the impact of nutrition supplementation on serum TMAO concentrations and found prebiotic supplementation resulted in a significant decrease in TMAO $(-0.237 \mu \mathrm{mol} / \mathrm{L}, P=.04)$. 


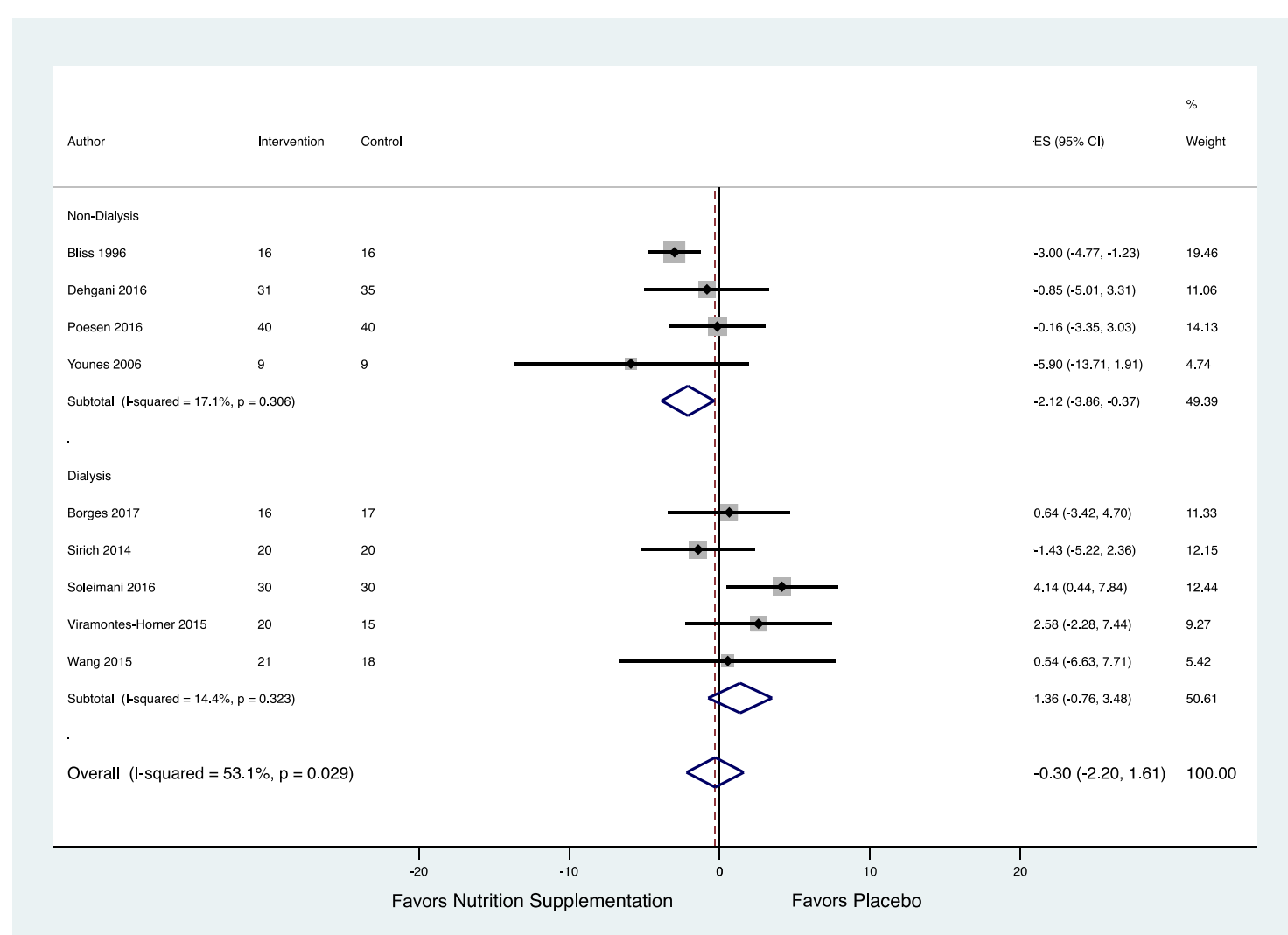

Figure 4. Effects of prebiotic, probiotic, and synbiotic supplementation on serum urea (mmol/L), by CKD stage. The means and SDs of changes from baseline are reported for trials. Effects of trials are presented as weights (percentages) and mean differences $(95 \% \mathrm{Cls})$. Weights are from random-effects analysis. Cls, confidence intervals; CKD, chronic kidney disease; IV, inverse variance; SDs, standard deviations.

The impact of nutrition supplementation on serum PAG was reported in 1 study. ${ }^{7}$ Prebiotic supplementation had no significant effect on serum PAG $(0.080 \mu \mathrm{mol} / \mathrm{L} P=.41)$.

\section{Microbiota Composition}

Two studies investigated the effect of nutrition supplementation on microbiota composition, both were synbiotic studies. ${ }^{9,25}$ Intervention duration ranged from 6 to 8 weeks, with 18-31 participants. Synbiotic formulation differed between the 2 studies (Table 1). Both studies noted significant increases in Bifidobacterium $\left(3.2 \% P=.003^{9}\right.$ and $4.2 \pm 0.88 \log _{10}$ cells/g to $5.5 \pm 1.72 \log _{10}$ cells $/ g$ $P=.034^{25}$ ) with one study also finding a significant increase in Lachnospiraceae $(2.1 \% P=.01)$, a nonsignificant increase in Lactobacillus $(0.7 \% P=.36)$, and a significant decrease in Ruminococcaceae $(4.3 \% P=.01){ }^{9}$

\section{Clinical Markers}

Few studies reported on change in clinical markers with prebiotic, probiotic, and/or synbiotic supplementation. Blood glucose levels were reported in 2 studies $^{7,27}$ and weight in 7 studies, $7,18,19,23,27-29$ while waist circumference was not reported in any included studies. It was uncertain whether prebiotic or probiotic supplementation improved blood glucose levels as the certainty of this evidence was very low (2 studies, 100 participants: $\mathrm{MD}-8.19 \mathrm{md} / \mathrm{dL}, 95 \% \mathrm{CI}-32.45$ to $\left.16.07, P=.51, \mathrm{I}^{2}=80 \%\right)^{7,27}$ (Supplementary Fig. 5). Heterogeneity was not able to be explained through sensitivity analyses. Compared with placebo, prebiotic, probiotic, and/or synbiotic supplementation may have had little or no impact on weight $(7$ studies, 357 participants: MD $0.40 \mathrm{~kg}, 95 \% \mathrm{CI}-0.95$ to $1.74, P=.56, \mathrm{I}^{2}=0 \%$; low certainty evidence) $)^{7,18,19,23,27-29}$ (Supplementary Fig. 6).

\section{Cardiovascular Risk}

Few studies reported on the effect of prebiotic, probiotic, and/or synbiotic supplementation on cardiovascular risk. Blood lipids were reported in 3 studies. ${ }^{27-29}$ It was uncertain whether nutrition supplementation improved total cholesterol (3 studies, 226 participants: MD $-6.23 \mathrm{mg} / \mathrm{dL}, 95 \%$ CI -25.86 to $13.41, P=.53$, $\left.\mathrm{I}^{2}=76 \%\right)$, low-density lipoprotein cholesterol (3 studies, 226 participants: $\mathrm{MD}-5.50 \mathrm{mg} / \mathrm{dL}, 95 \% \mathrm{CI}-15.54$ to $4.54, P=.28, \mathrm{I}^{2}=83 \%$ ), or triglyceride levels (3 studies, 226 participants: $\mathrm{MD}-1.57 \mathrm{mg} / \mathrm{dL}, 95 \% \mathrm{CI}-22.56$ to $\left.19.42, P=.88, \mathrm{I}^{2}=0 \%\right)$ as the certainty of this evidence was very low (Supplementary Figs. 7, 8, and 9). Heterogeneity was not able to be explained through sensitivity 
analyses. Data on left ventricular mass index, diastolic function, and blood pressure were not reported in any included studies.

\section{Infections}

None of the included studies reported an infection as an adverse event. One study reported on antibiotic use throughout the intervention. ${ }^{9}$ Patients who did not receive antibiotics during the study had marked reductions in IS $(-5 \mu \mathrm{mol} / \mathrm{L}, 95 \% \mathrm{CI}-8$ to $-1 \mu \mathrm{mol} / \mathrm{L}, P=.03)$ and PCS $(-25 \mu \mathrm{mol} / \mathrm{L}, 95 \%$ CI -38 to $-12 \mu \mathrm{mol} / \mathrm{L}$, $P=.001)$ compared with those who received antibiotics. Antibiotic use preceding baseline visit was an exclusion criterion in 6 of the 16 studies. ${ }^{7-9,19,27,30}$

\section{Adverse Events}

Three studies provided data on adverse events. ${ }^{9,21,26}$ One study reported initial hospitalization for 6 patients; 3 during washout, 2 during placebo, and 1 during synbiotic periods. ${ }^{9}$ Two studies each had one patient die of a myocardial infarct during the study period, both of which were deemed unrelated to the study intervention. ${ }^{21,26}$ None of the included studies reported on progression to ESKD as an outcome measure.

\section{Patient-Centered Outcomes}

Fecal characteristics were reported in 4 of the included studies. ${ }^{7,18,20,23}$ Two studies used the Bristol Stool Scale to measure stool consistency, with both reporting no effect after prebiotic and synbiotic supplementation. ${ }^{7,20}$ Stool frequency was reported in 2 studies with no effect after synbiotic supplementation $(P=.92)^{7}$ or prebiotic supplementation $(P=.48) .{ }^{18}$ Younes et $\mathrm{al}^{23}$ noted an increase in stool weight after prebiotic supplementation $(P<.05)$.

Gastrointestinal tolerance was investigated using a variety of measures: Gastrointestinal Symptom Rating Scale, ${ }^{9,25}$ other patient-reported questionnaires, ${ }^{7,20,28}$ and through patient interviews. ${ }^{8,18,22,27}$ No significant change in Gastrointestinal Symptom Rating Scale scores were noted after synbiotic supplementation $\left(P=.168^{9}\right.$ and $\left.P=.72^{25}\right)$. An increase in flatulence was reported in 2 prebiotic interventions, ${ }^{7,18}$ while improvements in bloating $(P \leq .05)$ and constipation $(P \leq .05)$ were noted after synbiotic supplementation. ${ }^{28}$

Colonic transit time was not reported in any of the included studies.

A variety of measures were used to assess adherence to treatment, with pill count being the most common. ${ }^{8,9,24,26-28}$ Overall adherence to nutrition supplementation was high, ranging from $82 \%$ to $97 \%$. $^{7,9,24,26,27}$

Health-related quality of life (QoL) was assessed using a range of tools (Short Form-36, ${ }^{9,26}$ Kidney Disease Quality of Life Short Form-36 ${ }^{8}$ and using a 10-point scale $\left.^{21,22}\right)$. No significant change to QoL was found in 3 studies, ${ }^{8,9,26}$ while one study found $85 \%(P>.05)$ of participants had a perceived higher QoL during the intervention period. ${ }^{22}$

\section{Discussion}

This systematic review assessed the effectiveness of prebiotic, probiotic, and/or synbiotic supplementation in adults with CKD. In mostly low certainty evidence, pooled results indicated that nutrition supplementation may have had little or no effect on eGFR, serum creatinine, serum urea, serum IS, serum PCS, blood glucose levels, weight, total cholesterol, low-density lipoprotein cholesterol, highdensity lipoprotein cholesterol, or triglyceride levels. Prebiotic supplementation may have slightly reduced serum urea concentrations $(-2.23 \mathrm{mmol} / \mathrm{L} P=.006)$, although there was no appreciable effect with probiotic or synbiotic supplementation. In addition, there may have been a positive benefit with all 3 types of nutrition supplementation (prebiotics, probiotics, and synbiotics) on serum urea concentrations $(-2.12 \mathrm{mmol} / \mathrm{L} P=.02)$ in nondialysisdependent CKD, however not to those who were dialysis dependent.

The reduction in serum urea with prebiotic supplementation is supported by the results of an earlier systematic review which found that dietary fiber intake was associated with significant reductions in serum urea (MD $-1.76 \mathrm{mmol} / \mathrm{L}$ [95\% CI, -3.00 to -0.51$]$, $P<.01)$ in $\mathrm{CKD}^{31}$ Reducing serum urea concentrations in CKD may be of importance as the diffusion of urea into the gut lumen favors the growth of intestinal bacteria that express urease as well as those that produce uremic toxins. Increased urease expression promotes the degradation of urea leading to the erosion of the epithelial barrier, thereby promoting systemic inflammation. ${ }^{32,33}$ The low certainty of effect of prebiotic, probiotic, and synbiotic supplementation on the uremic toxins, IS, and PCS, reflects the conflicting findings found in individual studies with increases, decreases, and neutral effects reported in the literature. ${ }^{10-12}$ Study design was likely an important limiting factor underpinning the disparate findings, given the short intervention durations (4-12 weeks), small participant numbers, ${ }^{31-40}$ and variable product formulations in these studies.

Microbiota composition was investigated in 2 studies, with synbiotic supplementation leading to higher abundances of Bifidobacterium ${ }^{9,25}$ and Lachnospiraceae ${ }^{9}$ and a decrease in Ruminococcaceae. Although the significant change in microbiota composition is encouraging, it is important to note that product formulation and dosage differed between the 2 studies, and only one study considered the potential modifying effects of participants' baseline microbiota profiles. ${ }^{9}$ Moreover, both studies used fecal samples as a surrogate for gut microbiota composition, even though this may not have accurately reflected the colonic mucosal microbiota. ${ }^{34}$ 
Prebiotic, probiotic, and synbiotic supplementation appeared to be well tolerated with few gastrointestinal side effects. In conjunction with a high rate of patient adherence, few adverse events and no decrease in health-related QoL, this preliminary, low certainty evidence suggested that prebiotic, probiotic, and synbiotic supplementation may be safe, well-tolerated, and acceptable forms of supplementation within the CKD population.

To our knowledge, this is the first systematic review investigating the effects of prebiotic, probiotic, and/or synbiotic supplementation on clinical and patient-centered outcomes in the CKD population. The strengths of this study included its robust design and comprehensive search strategy. However, it is acknowledged that this study had some limitations. Only a limited number of studies reported the primary outcome of kidney function and damage. The wide variability in product formulations further limited comparisons between interventions and measured outcomes. Moreover, some studies possibly provided inadequate amounts of product and/or an insufficient duration of treatment (2-24 weeks) to elicit a therapeutic response. ${ }^{35}$ The prebiotic studies evaluated used dosages ranging from 10 to $50 \mathrm{~g} /$ day, while the synbiotic studies used significantly lower dosages of prebiotic (2.3-15 g/day). A prebiotic dose of $>5 \mathrm{~g} /$ day has previously been shown to influence microbiota diversity, while a threshold dose of $15-20 \mathrm{~g} /$ day may be required to reduce uremic toxin concentrations. ${ }^{36}$ The included probiotic studies contained an array of strains and dosages. In other chronic diseases, the therapeutic response to probiotics has been strain specific, ${ }^{37}$ indicating that a specific mix of bacterial genera and dosages may be required for the CKD population. The responses to prebiotic, probiotic, and synbiotic supplementation may also have been influenced by a number of within-study factors, such as antibiotic use, ${ }^{38}$ washout periods, ${ }^{39}$ and dietary intake. $^{40,41}$ Antibiotic use throughout the intervention period was only documented in one study. ${ }^{9}$ A number of studies either did not describe or did not include a washout period, ${ }^{18,21-23}$ potentially confounding the results. Few studies included in this review controlled for potential dietary confounders. Finally, when applying the GRADE assessment, most studies were rated as "moderate" to "very low" quality thereby reducing confidence that the observed effect size was representative of the true effect.

\section{Practical Applications}

At this time, there is limited evidence to support the use of prebiotic, probiotic, and synbiotic supplementation in CKD. However, the observed reduction in serum urea in nondialysis-dependent CKD patients is promising. Owing to the small number of available studies it is premature to conclude whether one type of nutrition supplementation is superior to another at this time. Further well-designed interventions are required to establish the most appropriate supplementation formulation and its influence on patientlevel outcomes.

\section{Acknowledgments}

The authors thank Justin Clark for his assistance in the development of the search strategy.

Author Contributions: The author's responsibilities were as follows: C.M. assisted in the conceptualization of the review, conducted the initial literature search, performed eligibility screening and data extraction, assessed the risk of bias, conducted the analysis, drafted the manuscript, and had primary responsibility for the final content; C.I.R. performed eligibility screening and data extraction, assessed the risk of bias, interpreted the data, and critically revised the manuscript. D.W.J. assisted in the conceptualization of the review, provided methodological and clinical expertise, assisted in the analysis interpretation, and critically revised the manuscript. K.L.C. conceived the review, provided methodological and clinical expertise, assisted in analysis interpretation, and critically revised the manuscript; all authors read and approved the final manuscript.

\section{Supplementary Data}

Supplementary data related to this article can be found at https://doi.org/10.1053/j.jrn.2018.08.008.

\section{References}

1. Bello AK, Levin A, Tonelli M, et al. Assessment of Global kidney health Care status. JAMA. 2017;317:1864-1881.

2. Chadban SJ. Prevalence of kidney damage in Australian adults: the AusDiab kidney study. J Am Soc Nephrol. 2003;14:131S-138S.

3. Weiner DE. Chronic kidney disease as a risk factor for cardiovascular disease and all-Cause mortality: a pooled analysis of Community-Based studies. J Am Soc Nephrol. 2004;15:1307-1315.

4. Stenvinkel P, Carrero JJ, Axelsson J, Lindholm B, Heimburger O, Massy Z. Emerging biomarkers for evaluating cardiovascular risk in the chronic kidney disease patient: how do new pieces fit into the uremic puzzle? Clin J Am Soc Nephrol. 2008;3:505-521.

5. Moradi H, Sica DA, Kalantar-Zadeh K. Cardiovascular burden associated with uremic toxins in patients with chronic kidney disease. Am J Nephrol. 2013;38:136-148.

6. Vaziri ND, Wong J, Pahl M, et al. Chronic kidney disease alters intestinal microbial flora. Kidney Int. 2013;83:308-315.

7. Poesen R, Evenepoel P, de Loor H, et al. The influence of prebiotic Arabinoxylan Oligosaccharides on microbiota derived uremic Retention solutes in patients with chronic kidney disease: a randomized controlled trial. PLoS One. 2016;11:e0153893.

8. Sirich TL, Plummer NS, Gardner CD, Hostetter TH, Meyer TW. Effect of increasing dietary fiber on plasma levels of colon-derived solutes in hemodialysis patients. Clin J Am Soc Nephrol. 2014;9:1603-1610.

9. Rossi M, Johnson DW, Morrison M, et al. Synbiotics Easing renal failure by improving gut Microbiology (SYNER GY): a randomized trial. Clin J Am Soc Nephrol. 2016;11:223-231.

10. Meijers BK, De Preter V, Verbeke K, Vanrenterghem Y, Evenepoel P. p-Cresyl sulfate serum concentrations in haemodialysis patients are reduced by the prebiotic oligofructose-enriched inulin. Nephrol Dial Transplant. 2010;25:219-224.

11. Salmean YA, Segal MS, Palii SP, Dahl WJ. Fiber supplementation lowers plasma p-cresol in chronic kidney disease patients. I Ren Nutr. 2015;25:316-320.

12. Taki K, Takayama F, Niwa T. Beneficial effects of Bifidobacteria in a gastroresistant seamless capsule on hyperhomocysteinemia in hemodialysis patients. J Ren Nutr. 2005;15:77-80.

13. Moher D, Liberati A, TetzlaffJ, Altman DG, Group P. Preferred reporting items for systematic reviews and meta-analyses: the PRISMA statement. BMJ. 2009;339:b2535. 
14. Kidney Disease: Improving Global Outcomes (KDIGO) CKD Work Group. KDIGO 2012 clinical Practice guideline for the evaluation and management of chronic kidney disease. Kidney Int. 2013;3:1-150.

15. Wishart DS, Tzur D, Knox C, et al. HMDB: the human Metabolome Database. Nucleic Acids Res. 2007;35:D521-D526.

16. Higgins JPT, Green S. Cochrane Handbook for Systematic Reviews of Interventions Version 5.1.0. Available at: http://handbook.cochrane.org. Accessed June 2018.

17. Schünemann HBJ, Guyatt G, Oxman A, eds. GRADE Handbook for Grading Quality of Evidence and Strength of Recommendations. Available at: guidelinedevelopment.org/handbook. Accessed June 2018.

18. Bliss DZ, Stein TP, Schleifer CR, Settle RG. Supplementation with gum Arabic fiber increases fecal nitrogen excretion and lowers serum urea nitrogen concentration in chronic renal failure patients consuming a lowprotein diet. Am J Clin Nutr. 1996;63:392-398.

19. Dehghani HHF, Mozaffari-Khosravi H, Nouri-Majelan N, Dehghani A. Synbiotic supplementations for Azotemia in patients with chronic kidney disease: a randomized controlled trial. Iran J Kidney Dis. 2016;10:351-357.

20. Guida B, Germanò R, Trio R, et al. Effect of short-term synbiotic treatment on plasma p-cresol levels in patients with chronic renal failure: a randomized clinical trial. Nutr Metab Cardiovasc Dis. 2014;24:1043-1049.

21. Ranganathan N, Friedman EA, Tam P, Rao V, Ranganathan P, Dheer R. Probiotic dietary supplementation in patients with stage 3 and 4 chronic kidney disease: a 6-month pilot scale trial in Canada. Curr Med Res Opin. 2009;25:1919-1930.

22. Ranganathan NRP, Friedman EA, Joseph A, et al. Pilot study of probiotic dietary supplementation for promoting healthy kidney function in patients with chronic kidney disease. Adv Ther. 2010;27:634-647.

23. Younes H, Egret N, Hadj-Abdelkader M, et al. Fermentable carbohydrate supplementation alters nitrogen excretion in chronic renal failure. J Ren Nutr. 2006;16:67-74.

24. Borges NA, Carmo FL, Stockler-Pinto MB, et al. Probiotic supplementation in chronic kidney disease: a double-blind, randomized, placebocontrolled trial. J Ren Nutr. 2017;28:28-36.

25. Cruz-Mora J, Martinez-Hernandez NE, Martin del Campo-Lopez F, et al. Effects of a symbiotic on gut microbiota in Mexican patients with end-stage renal disease. J Ren Nutr. 2014;24:330-335.

26. Natarajan R, Pechenyak B, Vyas U, et al. Randomized controlled trial of strain-specific probiotic formulation (Renadyl) in dialysis patients. Biomed Res Int. 2014;2014:568571.

27. Soleimani A, Zarrati Mojarrad M, Bahmani F, et al. Probiotic supplementation in diabetic hemodialysis patients has beneficial metabolic effects. Kidney Int. 2016;91:435-442.

28. Viramontes-Horner D, Marquez-Sandoval F, Martin-del-Campo F, et al. Effect of a symbiotic gel (Lactobacillus acidophilus + Bifidobacterium lactis + inulin) on presence and severity of gastrointestinal symptoms in hemodialysis patients. J Ren Nutr. 2015;25:284-291.

29. Xie LM, Ge YY, Huang X, Zhang YQ, Li JX. Effects of fermentable dietary fiber supplementation on oxidative and inflammatory status in hemodialysis patients. Int J Clin Exp Med. 2015;8:1363-1369.

30. Wang IK, Wu YY, Yang YF, et al. The effect of probiotics on serum levels of cytokine and endotoxin in peritoneal dialysis patients: a randomised, double-blind, placebo-controlled trial. Benef Microbes. 2015;6: $423-430$

31. Chiavaroli L, Mirrahimi A, Sievenpiper JL, Jenkins DJ, Darling PB. Dietary fiber effects in chronic kidney disease: a systematic review and metaanalysis of controlled feeding trials. Eur J Clin Nutr. 2015;69:761-768.

32. Vaziri ND, Yuan J, Norris K. Role of urea in intestinal barrier dysfunction and disruption of epithelial tight junction in chronic kidney disease. Am J Nephrol. 2013;37:1-6.

33. Vaziri ND, Goshtasbi N, Yuan J, et al. Uremic plasma impairs barrier function and depletes the tight junction protein constituents of intestinal epithelium. Am J Nephrol. 2012;36:438-443.

34. Zoetendal EG, von Wright A, Vilpponen-Salmela T, Ben-Amor K, Akkermans ADL, de Vos WM. Mucosa-associated bacteria in the human gastrointestinal Tract are Uniformly Distributed along the colon and differ from the Community Recovered from Feces. Appl Environ Microbiol. 2002;68:3401-3407.

35. Vaziri ND. Effect of synbiotic therapy on gut-derived uremic toxins and the intestinal microbiome in patients with CKD. Clin J Am Soc Nephrol. 2016;11:199-201.

36. Rossi M, Klein K, Johnson DW, Campbell KL. Pre-, pro-, and synbiotics: do they have a role in reducing uremic toxins? A systematic review and meta-analysis. Int J Nephrol. 2012;2012:673631.

37. Singh S, Stroud AM, Holubar SD, Sandborn WJ, Pardi DS. Treatment and prevention of pouchitis after ileal pouch-anal anastomosis for chronic ulcerative colitis. Cochrane Database Syst Rev. 2015:CD001176.

38. Backhed F, Fraser CM, Ringel Y, et al. Defining a healthy human gut microbiome: current concepts, future directions, and clinical applications. Cell Host Microbe. 2012;12:611-622.

39. Haller D, Antoine JM, Bengmark S, Enck P, Rijkers GT, LenoirWijnkoop I. Guidance for substantiating the evidence for beneficial effects of probiotics: probiotics in chronic inflammatory bowel disease and the functional disorder irritable bowel syndrome. J Nutr. 2010;140:690S-697S.

40. Rossi M, Johnson DW, Xu H, et al. Dietary protein-fiber ratio associates with circulating levels of indoxyl sulfate and p-cresyl sulfate in chronic kidney disease patients. Nutr Metab Cardiovasc Dis. 2015;25:860865.

41. Kalantar-Zadeh K, Kopple JD, Deepak S, Block D, Block G. Food intake characteristics of hemodialysis patients as obtained by food frequency questionnaire. J Ren Nutr. 2002;12:17-31. 